\title{
Combined Disruption Of The Thoracic Spine And Costal Arch Fracture: An Indicator Of A Severe Chest Trauma
}

\section{Stefan Schulz-Drost}

Helios Hospital Schwerin

\section{Stephan Kloesel}

Helios Hospital Schwerin

Jan Carsten Kühling

Helios Hospital Schwerin

\section{Axel Ekkernkamp}

Medical University Greifswald

M. Sinan Bakir ( $\nabla$ sinan.bakir@uni-greifswald.de )

Medical University Greifswald

\section{Research Article}

Keywords: Thoracic spine fracture, costal arch fracture, severely injured, indicator injury, intercostal hernia.

Posted Date: February 15th, 2022

DOI: https://doi.org/10.21203/rs.3.rs-1047361/v2

License: (c) (i) This work is licensed under a Creative Commons Attribution 4.0 International License. Read Full License 


\section{Abstract}

Background: Blunt high-energy trauma increase the risk of associated thoracic and accompanying abdominal organ injuries in chest trauma. A higher overall injury severity has been shown for sternovertebral injuries caused by a flexion-compression mechanism. Literature for a hyperextensiondistraction mechanism equivalent to a costal arch fracture combined with a thoracic spinal fracture is sparse. Therefore, we want to show the clinical significance of this entity.

Methods: $A$ retrospective analysis of our level-one trauma center collective of severely injured patients was performed. Patients from 2013 to 2019 that involved an Injury Severity Score (ISS) $\geq 16$ were screened for the combined thoracovertebral injury entity and analyzed concerning its accident mechanism, the injury spectrum, and the clinical course.

Results: The thoracovertebral injury entity is rare. Three male patients (50-65 years old) with ISS of 57, 41 , and 20 could be observed from over 1000 severely injured. All patients suffered from high-energy trauma and from tearing of the thoracic vertebrae regions 7-9. The costal arch was torn in the anterior area. All cases showed a posterolateral serial rib fracture, a traumatic intercostal pulmonary hernia, and an avulsion of the diaphragm. Two patients sustained further life-threatening injuries, such as severely bleeding avulsion to the azygos vein and a rupture of the spleen. The physicians performed exploratory thoracotomy, closure of the pulmonary hernia, re-fixation of the diaphragm and angle-stable plate osteosynthesis of the fractured ribs, including the costal arch. The spine was fixed dorsally using a screwrod system. The patients were discharged home with independent mobility and without neurological damage.

Conclusions: This thoracovertebral injury entity is associated with high overall injury severity and lifethreatening thoracoabdominal injuries. Injuries to the costal arch can act as an indicator of severe trauma. They should be detected thorough clinical examination and assessment of the trauma CT in the soft tissue window.

\section{Background}

Blunt trauma mechanisms dominate the causes of thoracic trauma and thoracolumbar abdominal trauma $(1,2)$. Over $95 \%$ of the severely injured were harmed by a blunt trauma mechanism $(1,3)$. Thoracic trauma remains the most common injury to the organ cavities, occurring in just under half of all seriously injured people (4). Simultaneously, the abdomen was involved in only around $16 \%$ of all polytrauma cases $(1,3)$.

High-speed accidents - where the accident mechanisms act on the trunk with high energy - are the most feared. These multi-structural injuries often cause a high risk of death (5). A similar connection is known and described for a sternovertebral injury when a thoracic vertebral fracture coincides with a sternal fracture $(6,7)$. These injuries are more frequently associated with accompanying thoracic injuries, such as lung contusion, cardiac contusion, pneumothorax, and hemothorax $(5,6,8)$. 
Injuries with the so-called hyperextension-distraction mechanism of the human trunk are considered to be particularly risky. Individual cases with a ruptured spinal fracture and an anterior rupture of the chest wall with torn sternum are described here (5). It can be assumed that these injuries are observed so rarely that most patients die at the accident scene. Serious organ injuries between the sternum and spine with the heart and large vessels' involvement can quickly lead to death.

A similar accident mechanism can also lead to a tearing of the thoracic wall and a simultaneous spinal column fracture with more frontal to side impacts. According to our hypothesis, the combined injury of the costal arch and spinal column fractures at the level of the thoracic spine could be an indicator of very severe trauma. The extent to which this injury entity is associated with severe accompanying injuries has hardly been researched. This retrospective analysis aims to examine and discuss the clinical significance of a thoracic vertebra disruption in combination with a costal arch fracture.

\section{Methods}

The data of this survey are based on retrospective analyzes of our in-house collective of severely injured patients in a level-one trauma center in Germany. Patient data from 2013 to 2019 that involved an Injury

Severity Score (ISS) of $\geq 16$ and a primary admission to our trauma center via the emergency department (ED) were included. We have limited the collective to severely injured patients with ISS $>16$, since a distraction fracture of a vertebral body corresponds to at least Abbreviated Injury Scale (AIS) of 3 and in combination with a relevant chest trauma (AIS $\geq 3$ ) this automatically results in at least ISS of 16 . Another inclusion criterion was the presence of a high-energy trauma (fall from $>3 \mathrm{~m}$ height, accident by car or motorcycle, miscellaneous). Exclusion criteria consisted of several parameters: age $<18$ years, thoracic injuries with an AIS less than 3 , and early transfer to another hospital (since no outcome would be available) (9).

Among the included patients, those suffering from severe combination injuries to the trunk were identified. The identified patients' files were examined, and their medical history, injury morphology, and outcome data were gathered. The overall injury severity was assessed using the ISS (10).

All patients, or a legal guardian, gave their informed written consent for collecting and anonymously publishing data. Due to the retrospective character of the analysis and the given informed consent, no additional approval from the local ethics committee was necessary (Ethics Committee of the University Hospital Erlangen). Due to the small number of cases, no further statistical analysis was performed.

\section{Results}

Out of $n=1012$ severely injured with ISS $>16$, three striking cases could be identified who suffered a combination with a fracture of the thoracic spine and a costal arch fracture after high-energy trauma. These patients were all male $(50,58$, and 65 years old). They all suffered serious overall injury patterns 
with ISSs of 57,41 , and 20 points. The first patient was buried by soil. High velocity traffic accident with a $\operatorname{car}(100 \mathrm{~km} / \mathrm{h})$ and a fall from a great height $(6 \mathrm{~m})$ were specified as the other accident mechanisms.

The disruption of the thoracic spine affected thoracic vertebrae with one fracture in between the 7th and the 9th level in all cases. The costal arch was monolaterally torn in the anterior area with fractures of the ribs VII and VIII at their conjunction of the cartilage (two cases on the right site). All cases showed an additional ipsilateral rib fractures series posterolateral with involvement at least of the ribs VII-IX (Case 1: III-X; Case 2: VII-IX; Case 3: VI-X), a traumatic intercostal pulmonary hernia requiring intervention, and an avulsion of the diaphragm (Fig. 1). Also, both the first and second patients sustained other lifethreatening injuries.

\section{Case 1}

also had severe tears of the right lower lobe of the lung and a heavily bleeding avulsion injury to the azygos vein near the thoracic vertebral disruption, as well as blunt abdominal trauma. The blood leaked freely into the pleural cavity, creating a massive hemothorax. A short time later, the patient was required to be resuscitated and could be surgically resuscitated as part of an emergency thoracotomy. After hemostasis, the thorax was closed according to damage control principles, and then the definitive surgical intervention was carried out in stages.

\section{Case 2}

had a rupture of the anterior parasternal chest wall with fractures of the costal cartilages $3-8$, an open, torn epigastric abdominal wall with evisceration, and a rupture of the spleen.

Therapeutically, in all cases, the physicians performed a multistage procedure with the focus on damage control surgery as first. Thus, Case 1 received emergency thoracotomy for hemorrhage control followed by urgent laparotomy which has also been done in Case 2. The severe rupture of the diaphragm had been fixed in both cases during this procedure in a supine position. Once the patient had been stabilized via intensive care treatment, the next step was the fixation of the vertebral spine in a prone position by a screw and rod system. Operative treatment had been completed by second look thoracotomy in a lateral decubitus position. Within this procedure, surgical stabilization of rib fractures (SSRF; plates and locked screws) had been included as well as the closure of the intercostal hernia by direct suture of the intercostal muscle layers (Fig. 2).

The neurological outcome of the patients showed good results without any relevant neurological damage. Patients 1 and 2 had to be ventilated (prolonged) and tracheostomized. They were transferred to a weaning clinic on days 41 and 23 and were discharged home with independent mobility and spontaneous breathing after 3 and 4 months, respectively. Patient 3 was able to receive follow-up rehabilitation treatment right after the stay in our clinic. In this way, the first and third patients could be rehabilitated back to work (5 point at Glasgow Outcome Scale). The second patient was rehabilitated for 
independent everyday mobility and daily life activities but had a limited capacity due to bilateral chest trauma and could not return to work (4 point at Glasgow Outcome Scale).

\section{Discussion}

This study indicates that hyperextension fractures of the thoracic spine may result in high injury severity and overall life-threatening thoracoabdominal injuries once the lower ribs at the costal margin show a fracture at the same time. As suspected, injuries to the costal arch can act as an indicator of severe trauma, especially in this context.

Caused by high-energy accidents, all of our patients suffered a hyperextension fracture of the central thoracic spine in the thoracic vertebrae regions 7-9. The energy apparently continued over the intercostal space to the costal arch. The intercostal space tore together with the thoracic spine; the energy was diverted in the costal arch between levels 7 and 9, i.e., at the ribs' point of confluence. This created an unstable situation for the anterior and lateral chest wall. This injury pattern is analogous to the one described in the literature in the case of a rupture of the spinal column with an anterior rupture of the chest wall due to the tearing of the sternum (5-7). In our three cases, the rupture of the intercostal space resulted in a herniation of the lung parenchyma into the chest wall. In each case, the diaphragm was torn out at the costal arch to create a two-cavity injury.

Although two patients suffered further life-threatening injuries, which had to be addressed as an emergency, the outcomes were good. The only patient who was unable to work again had a bilateral chest trauma with remaining limited capacity. It should be noted here that the left operated side showed a significantly better function than the conservatively treated right side, which initially appeared to be less severely injured. We see this as a supporting argument for our treatment recommendation that surgical stabilization of the thorax should be generously indicated for this severe combined injury entity. Although the literature remains somehow controversial to surgical repair of rib fractures in general, we believe that there are significant advantages to surgical intervention, such as reduced respiratory failure (11-14). Recent studies support our estimation of a beneficial operative therapy strategy, especially in analogous severe thoracic traumata as in flail chest or costoclavicular injuries $(11,15-22)$. For other, similar injury patterns with e.g. transdiaphragmatic intercostal hernia and other costal margin injuries, equivalent therapeutic strategies as in our cases were used (23-25).

The peculiarity of the injury combination of a distraction injury to the thoracic spine plus a fracture of the costal arch is above all the risk of two major accompanying injuries: an intercostal tear with consecutive lung herniation and a diaphragmatic tear leading to a consecutive two-cavity injury. The indicator function for a severe trauma is again underlined: despite the severity of the associated injuries, two-cavity traumata and particularly diaphragmatic injuries are often diagnosed only secondarily and delayed (23, 24).

An associated high ISS in case of an unstable costal arch injury combined with a distraction fracture of the thoracic spine could be attributed to the high injury severity of the thorax itself, since serial rib 
fractures in monolateral injuries are already related to AIS 3 and 4 (9). However, we were able to show that there are also other severe concomitant injuries that are of considerable importance for the overall injury severity. This is underlined by the high overall ISS values with 57,41 , and 20 points which is in the majority significantly higher than the inclusion criterion of ISS $\geq 16$.

Due to the low number of cases, the findings reported here should be interpreted cautiously. As in every retrospective evaluation, causal conclusions cannot be drawn. Since our selection criteria was ISS $\geq 16$ due to the high AIS values of the respective single injury, only severely injured patients were analyzed. Further research should investigate the relevance in low-energy accidents, e.g. in geriatric collectives, in order to double-check a possible underestimation in the frequency of this injury entity. Also, further prospective studies with larger sample sizes should attempt to validate our recommended therapeutic strategies. However, case-related clinical details can be analyzed and in each case history involving the fracture kinetic and morphological aspects, which is an advantage of our study in contrast to register studies with large cohorts.

\section{Conclusions}

Because of their association with high overall injury severity, injuries to the costal arch should be detected or excluded by thorough clinical examination and assessment of the trauma CT in the soft tissue window which makes the difference to fractures series of the ribs in their shaft segment. This clinical recommendation could lead to improved detection of associated life-threatening thoracoabdominal injuries.

In summary, during the initial physical examination, attention should be given to instabilities in the thorax, especially on the cartilaginous costal arch. If the radiologic imaging then shows a bony injury to the thoracic spine, there is a higher risk of concurrent intercostal hernia and diaphragmatic rupture in the sense of a two-cavity injury. In severe cases, life-threatening accompanying injuries can also be observed, which underlines the clinical significance of this injury entity. Thus, we confirmed our hypothesis that a hyperextension fracture of the thoracic vertebra plus costal arch fracture is an important indicator of very severe trauma.

\section{List Of Abbreviations}

Injury Severity Score - ISS

Abbreviated Injury Scale - AIS

Emergency Department - ED

Surgical Stabilization of Rib Fractures - SSRF

\section{Declarations}


Ethics approval and consent to participate All data were collected anonymously and the study has been performed in accordance with the ethical standards laid down in the 1964 Declaration of Helsinki and its later amendments. Due to the retrospective character of the analysis and the given informed consent, no additional approval from local ethics committee was necessary (University Hospital Erlangen, Ethics Committee).

Written informed consent to participate was obtained from all patients or a legal guardian.

Consent for publication All patients, their parents, or a legal guardian gave their informed written for collecting and anonymously publishing data.

Availability of data and materials All data generated or analysed during this study are included in this published article.

Competing interests The senior author S. Schulz-Drost is a member of the AO TK Thoracic Surgery Expert Group (THEG), he has a consultant agreement with DePuySynthes and he is working for the national DSTC $^{\text {TM }}$ program. The other authors M. Sinan Bakir, Stephan Kloesel, Jan Carsten Kühling and Axel Ekkernkamp state that they are not involved in any conflict of interest.

Funding No funding has been received in connection to this study.

Authors' contributions MSB and SSD had full access to all of the data in the study and take responsibility for the integrity of the data and the accuracy of the data analysis, including and especially any adverse effects. MSB, SK, JCK, AE and SSD contributed substantially to the study design, data analysis and interpretation, and the writing of the manuscript. All authors contributed to the critical revision and provided final approval of the manuscript.

\section{Acknowledgments}

We would like to show our gratitude to all participating patients for providing the data and giving informed consent for publication. No funds were received in connection to this study.

\section{Authors' information}

SSD is a member of the AO TK Thoracic Surgery Expert Group (THEG) and a member of the Chest Wall Injury Society (CWIS).

\section{References}

1. DGUß T. Jahresbericht 2019 - TraumaRegister DGU® [Annual report 2019 - TraumaRegister DGU®]. September 2019.

2. Schulz-Drost S, Krinner S, Langenbach A, Oppel P, Lefering R, Taylor D, et al. TraumaRegister DGU. Concomitant Sternal Fracture in Flail Chest: An Analysis of 21,741 Polytrauma Patients from the 
TraumaRegister DGUß. Thorac Cardiovasc Surg. 2017;65(7):551-9.

3. Schulz-Drost S, Oppel P, Grupp S, Krinner S, Langenbach A, Lefering R, et al. [Bony injuries of the thoracic cage in multiple trauma: Incidence, concomitant injuries, course and outcome]. Der Unfallchirurg. 2016;119(12):1023-30.

4. Galan G, Penalver JC, Paris F, Caffarena JM, Jr., Blasco E, Borro JM, et al. Blunt chest injuries in 1696 patients. Eur J Cardiothorac Surg. 1992;6(6):284-7.

5. Bailey J, Vanderheiden T, Burlew CC, Pinski-Sibbel S, Jordan J, Moore EE, et al. Thoracic hyperextension injury with complete "bony disruption" of the thoracic cage: Case report of a potentially life-threatening injury. World J Emerg Surg. 2012;7(1):14.

6. Fowler AW. Flexion-compression injury of the sternum. J Bone Joint Surg Br. 1957;39-B(3):487-97.

7. De Tarnowsky G. VII. Contrecoup Fracture of the Sternum. Ann Surg. 1905;41(2):252-64.

8. Ghent WR. Crush injuries to the chest. Can J Surg. 1959;2(3):265-71.

9. Kramer CF, Barancik JI, Thode HC, Jr. Improving the sensitivity and specificity of the abbreviated injury scale coding system. Public Health Rep. 1990;105(4):334-40.

10. Osler T, Baker SP, Long W. A modification of the injury severity score that both improves accuracy and simplifies scoring. J Trauma. 1997;43(6):922-5; discussion 5-6.

11. Langenbach A, Pinther M, Krinner S, Grupp S, Ekkernkamp A, Hennig FF, et al. Surgical Stabilization of Costoclavicular Injuries - A Combination of Flail Chest Injuries and a Clavicula Fracture. Chirurgia (Bucharest, Romania: 1990). 2017;112(5):595-606.

12. Marasco SF, Martin K, Niggemeyer L, Summerhayes R, Fitzgerald M, Bailey M. Impact of rib fixation on quality of life after major trauma with multiple rib fractures. Injury. 2019;50(1):119-24.

13. Griffard J, Daley B, Campbell M, Martins D, Beam Z, Rowe S, et al. Plate of ribs: single institution's matched comparison of patients managed operatively and non-operatively for rib fractures. Trauma Surg Acute Care Open. 2020;5(1):e000519.

14. Marro A, Chan V, Haas B, Ditkofsky N. Blunt chest trauma: classification and management. Emerg Radiol. 2019;26(5):557-66.

15. Schuurmans J, Goslings JC, Schepers T. Operative management versus non-operative management of rib fractures in flail chest injuries: a systematic review. Eur J Trauma Emerg Surg. 2017;43(2):1638.

16. Lodhia JV, Konstantinidis K, Papagiannopoulos K. Surgical management of multiple rib fractures/flail chest. J Thorac Dis. 2019;11(4):1668-75.

17. Majercik S, Pieracci FM. Chest Wall Trauma. Thorac Surg Clin. 2017;27(2):113-21.

18. Uchida K, Miyashita M, Kaga S, Noda T, Nishimura T, Yamamoto H, et al. Long-term outcomes of surgical rib fixation in patients with flail chest and multiple rib fractures. Trauma Surg Acute Care Open. 2020;5(1):e000546.

19. Divisi D, Mucilli F, Leonardo GD, Zaccagna G, De Vico A, Camplese P, et al. Plates versus struts versus an extracortical rib fixation in flail chest patients: Two-center experience. Injury. 2021;52(2):235-42. 
20. Fokin AA, Hus N, Wycech J, Rodriguez E, Puente I. Surgical Stabilization of Rib Fractures: Indications, Techniques, and Pitfalls. JBJS Essent Surg Tech. 2020;10(2):e0032.

21. Tignanelli CJ, Rix A, Napolitano LM, Hemmila MR, Ma S, Kummerfeld E. Association Between Adherence to Evidence-Based Practices for Treatment of Patients With Traumatic Rib Fractures and Mortality Rates Among US Trauma Centers. JAMA Netw Open. 2020;3(3):e201316.

22. Langenbach A, Krinner S, Hennig FF, Ekkernkamp A, Schulz-Drost S. [Injuries of the posterior and lateral chest wall-importance of an additional clavicular fracture]. Unfallchirurg. 2018;121(8):61523.

23. Sapp A, Nowack T, Benjamin Christie D. Transdiaphragmatic Intercostal Hernia After Trauma: A Case Report, Literature Review, and Discussion of a Challenging Clinical Scenario. Am Surg. 2020:3134820945273.

24. Gooseman MR, Rawashdeh M, Mattam K, Rao JN, Vaughan PR, Edwards JG. Unifying classification for transdiaphragmatic intercostal hernia and other costal margin injuries. Eur J Cardiothorac Surg. 2019;56(1):150-8.

25. Hruska LA, Corry D, Kealey GP. Transdiaphragmatic intercostal hernia resulting from blunt trauma: case report. J Trauma. 1998;45(4):822-4.

\section{Figures}
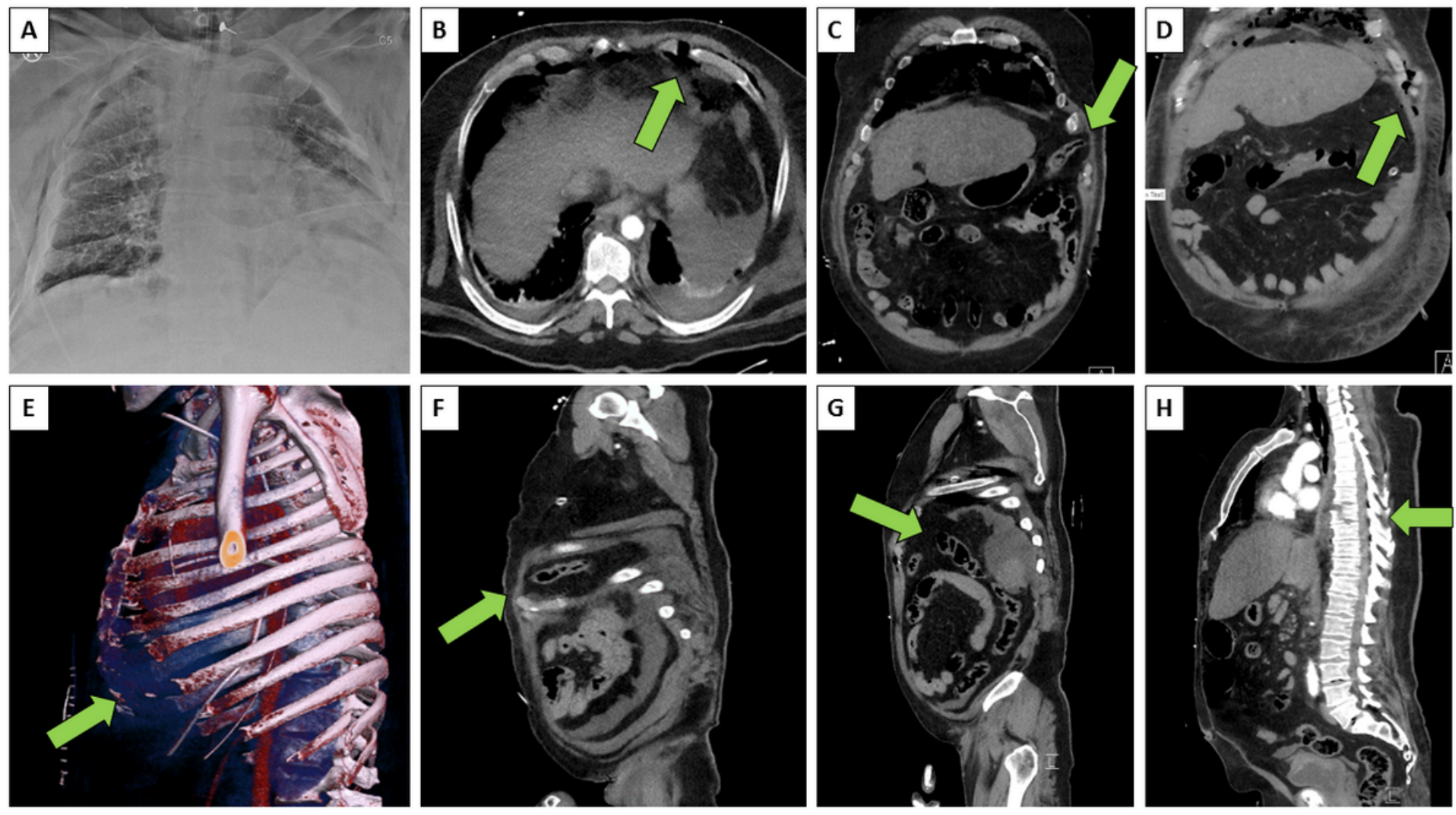

Figure 1 
Clinical example of combined disruption of the thoracic spine and costal arch fracture, preoperative. Initial $x$-ray of the chest shows left sided high riding diaphragm (A). CT scan proves anterior cartilage fracture (B), intercostal hernia within the widened $7^{\text {th }}$ intercostal space (C$F)$, disruption of the diaphragm (G), and distraction fracture of the $7^{\text {th }}$ thoracic vertebra $(H)$. The sternum, the mediastinal organs, the center of the diaphragm, and the great vessels did not show any injuries. The respective injuries are marked with arrows.
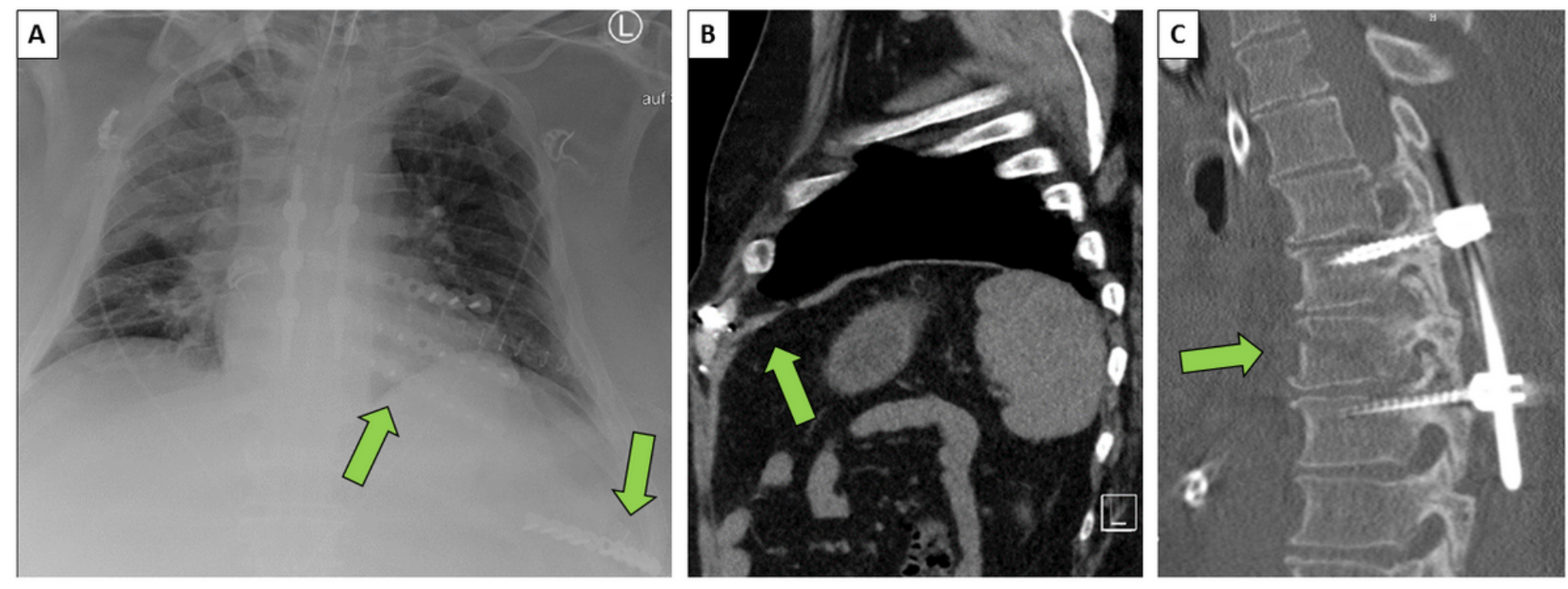

\section{Figure 2}

Clinical example of combined disruption of the thoracic spine and costal arch fracture, postoperative. Postoperative $x$-ray of the chest (A) and CT scan (B) show normal intercostal spaces and normal shape of the diaphragm. The lower costal margin has been fixed with a locking plate at the confluence of the $7^{\text {th }}$ and the $8^{\text {th }}$ rib. The thoracic spine shows anatomic alignment after dorsal instrumentation with an internal screw-rod-system (Viper, DePuySynthes, Switzerland). The respective injuries are marked with arrows. 